\title{
Concurrent Behcet'sDisease with Deep Venous Thrombosis of The Lower Extremity Alone: A Case Report
}

\author{
Hong Tao Xiong* \\ Department of Hand and Micro-Vascular Surgery, Shenzhen People's Hospital, (The Second Clinical Medical CollegeJinan University;The \\ First Affiliated Hospital, Southern University of Science and Technology) Shenzhen 518020, Guangdong,China \\ Corresponding author: Hong Tao Xiong, Department of Hand and Micro-Vascular Surgery, Shenzhen People's Hospital, (The Second \\ Clinical Medical Collegejinan University; The First Affiliated Hospital, Southern University of Science and Technology) Shenzhen \\ 518020, Guangdong, China
}

\section{ARTICLE INFO}

Received: 㹃 October 09, 2020

Published: October 19, 2020

\section{ABSTRACT}

Keywords: Behcet's Disease; Lower Extremity; Deep Venous Thrombosis; Diagnosis; Treatment

Citation: Hong Tao Xiong. Concurrent Behcet'sDisease with Deep Venous Thrombosis of The Lower Extremity Alone: A Case Report. Biomed J Sci \& Tech Res 31(2)-2020. BJSTR. MS.ID.005073.

\section{Introduction}

It is well known that deep vein thrombosis of the lower limbs is a common condition with a high incidence. Behcet's disease, on the other hand, is a rare disorder with a low incidence [1]. The author reviewed a patient who visited an outpatient clinic for follow-up of deep vein thrombosis in the lower extremities. After physical examination, detailed history acquisition and further testing, it was found that the patient suffered from a primary disorder, Behcet's disease, which presented with only deep vein thrombosis of the lower limbs, without involvement of the neurological, respiratory or digestive systems and vascular lesions elsewhere. After targeted treatment of rheumatology and immunology, the patient's condition showed marked improvement. Here, we present a summary of this case.

\section{Patient Description}

A 31-year-old man with the diagnosis of deep vein thrombosis in the lower extremities in June 2017 after a colour Doppler ultrasound examination revealed thrombosis of the lower femoral vein in both lower limbs. Blood tests showed a D-dimer level of
$921.20 \mathrm{ng} / \mathrm{ml}$, and a fibrinogen of $5.50 \mathrm{~g} / \mathrm{L}$. After the diagnosis of deep vein thrombosis in both lower extremities was made, a therapeutic dose of rivaroxaban (15 mg, twice a day) was given for 3 weeks, which was followed by a maintenance dose of rivaroxaban (20mg, once a day). Once the dose was reduced at one stage during treatment to $10 \mathrm{mg}$ once a day, the patient reported an obvious increase in leg swelling. In September of 2017, a thrombophilia screen proved negative results: Protein C $96.00 \%$, protein S activity 121.7\%, fibrinase (AT) 93.00\%, fibrinogen degradation products $0.51 \mathrm{ug} / \mathrm{ml}$, fibrinogen activity (PLG) 111\%, lupus anticoagulant negative (-) and LA Normalised Ratio (TR) 1.18. In July of 2018, the result of a repeat D-dimer test was $336.11 \mathrm{ng} / \mathrm{ml}$, which was within the normal reference range.

When the author first saw the patient in November 2017, a physical examination revealed mild swelling of the lower limbs and multiple red skin rashes (Figure 1). A repeat colour Doppler ultrasound showed thrombosis of the lower femoral vein and iliac vein in both lower limbs with partial recanalization.Since the patient had no obvious trigger for deep venous thrombosis in both lower 
limbs, and anticoagulant therapy did not achieve a satisfactory result, we delved deeper into the patient's medical history. It was found that the patient had 20-year history of recurrent oral ulcers with no clear cause and also history of ocular foreign body sensation. There was also 6-year history of nodular erythema in the lower limbs. Some of the nodules had become purulent and formed ulcers and crusts. The patient did not seek diagnosis and treatment for these symptoms, and the nodules healed by themselves, leaving behind pigmentation and scars. Superficial scrotal ulcers developed 5 years ago. These healed with the use of topical medication and did not recur. Intermittent interphalangeal joint pain in both hands as well as pain in the first metatarsophalangeal joint developed 3 years ago.
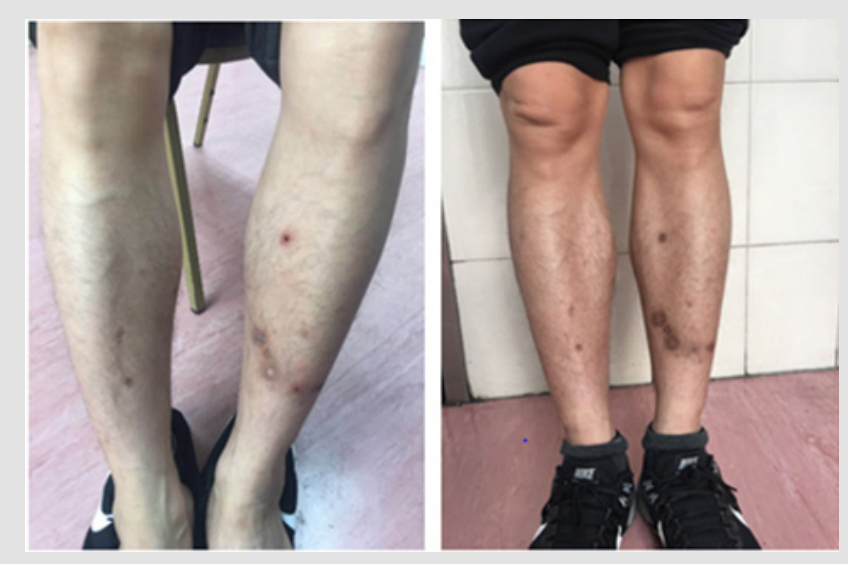

Figure 1: Bilateralcalf swelling and skinlesions in November 2017/ Physical examination after immunotherapy002E.

These symptoms resolved after the patient applied topical treatments. He reported that he had no visual impairment. After eye examination, it was found that both eyes had mild retinal vasculitis and vitreitis. The naked eye vision of both eyes was 4.8. There was no obvious history of abdominal pain, diarrhoea, or shortness of breath. The patient also denied any history of hypertension, diabetes, coronary heart disease, hepatitis, tuberculosis or other infectious disease. A single ultrasound examination of the inferior vena cava was performed, and no abnormalities were seen. Based on the patient's symptoms and treatments described above and coupled with the history of recurrent rashes of unknown cause in both calves, the possibility of a rheumatic immune condition was considered and a single biopsy was taken from a left calf skin lesion. The results showed: localised ulcers in the skin tissue sample, fibroid necrosis of the vascular wall in parts of the dermis, infiltration of lymphocytes, neutrophils and eosinophils in appendages and surrounding blood vessels, with possible vascular inflammatory changes. A review by the Department of Rheumatology and Immunology was sought, and the patient was admitted to the hospital with a diagnosis of "Behcet's disease." The case and characteristics were summarised in (Table 1).
Table 1: Summary of the case and characteristics/ findings.

\begin{tabular}{|c|c|}
\hline Case & Characteristics and findings \\
\hline Diagnosis & VTE, BD \\
\hline Age/sex/ethnicity & 31/M/Chinese \\
\hline Medical /smoking status & No Asthma or atopic diathesis, non-smoker \\
\hline $\begin{array}{l}\text { Characteristics of } \\
\text { vasculitis }\end{array}$ & Recurrent oral ulcers, genital ulcers \\
\hline $\begin{array}{l}\text { Dermatological } \\
\text { manifestations }\end{array}$ & Multiple skin ulcers on both legs \\
\hline \multirow{5}{*}{ Auxiliary Examination } & Cerebrovascular MR: negative \\
\hline & Chest CT: negative \\
\hline & $\begin{array}{l}\text { Thoracic and abdominal aorta enhanced } \\
\text { MR: negative }\end{array}$ \\
\hline & $\begin{array}{c}\text { Colour Doppler of inferior vena cava: } \\
\text { negative }\end{array}$ \\
\hline & $\begin{array}{l}\text { Doppler ultrasound: Recanalization of part } \\
\text { of deep vein in the lower extremity }\end{array}$ \\
\hline \multirow{9}{*}{ Lab results } & Immunoassay: lupus anticoagulant (LA): \pm \\
\hline & LA normalization ratio (TR): 1.48 \\
\hline & ANA: negative \\
\hline & ANCA: negative \\
\hline & CRP: $31.6 \mathrm{mg} / \mathrm{l}$ \\
\hline & ACE: $258.8 \mathrm{u} / \mathrm{l}$ \\
\hline & Vasculitis series: negative \\
\hline & anti-phospholipid antibody: negative \\
\hline & anti dsDNA: negative \\
\hline $\begin{array}{l}\text { Skin pathological } \\
\text { examination }\end{array}$ & $\begin{array}{c}\text { Cellulose-like necrosis in part of the } \\
\text { vascular wall of the dermis. Lymphocytes, } \\
\text { neutrophils, and eosinophils infiltration } \\
\text { in the appendages and around the blood } \\
\text { vessels }\end{array}$ \\
\hline Eye examination & $\begin{array}{l}\text { Both eyes had mild retinal vasculitis and } \\
\text { vitreitis. The naked eye vision of both eyes } \\
\text { was } 4.8 \text {. }\end{array}$ \\
\hline \multirow{5}{*}{ Treatment medications } & Rivaroxaban \\
\hline & Prednisone \\
\hline & Thalidomide \\
\hline & Diclofenac \\
\hline & Hydroxychloroquine Sulfat \\
\hline
\end{tabular}

Ulcers were visible on the tip of the tongue. There were no murmurs detected on auscultation of the neck. Both lungs were clear on auscultation, and there were no fine or coarse crackles heard. Heart rhythm was regular and there were no pathological murmurs heard in any of the cardiac auscultation areas. The abdomen was flat and soft, with no pain on palpation or rebound tenderness; there was no distension of the liver or spleen and no vascular bruits on auscultation. There was no swelling or tenderness in joints of the extremities and no tenderness in the muscles of the extremities. Muscle tone was normal. Infiltrating subcutaneous nodular ulcers 
that were red on the surface could be seen scattered on both lower limbs, along with old skin rashes and pigmentation.

Cerebrovascular magnetic resonance angiography (MRA) showed: 1. A few spotted demyelinating lesions under the bilateral frontal cortex; 2. No abnormalities were seen in cranial MRA and magnetic resonance venography (MRV). Enhanced chest and abdominal aorta MRA showed no obvious abnormalities. Chest CT scan also showed no abnormalities. Lupus anticoagulant testing showed: LA normalized ratio (TR) 1.48, Lupus anticoagulant weakly positive $( \pm)$. High-sensitivity C-reactive protein $31.6 \mathrm{mg} / \mathrm{L}$ (immunoturbidimetric method), angiotensin converting enzyme 258.8U/L (rate method), vasculitis series (-), ANA series (-), antiphospholipid antibody series (-). Anti-double-stranded DNA $(-)$. Stool and urine samples normal (-), faecal occult blood test (-). Pathological examination of the skin of the left calf showed fibroid necrosis in the vascular wall of the dermis, and infiltration of lymphocytes, neutrophils, and eosinophils around the appendages and blood vessels.

Consider vasculitis. There was no history of food or drug allergies and the patient did not drink alcohol or smoke. There was no family history of similar symptoms. The final diagnosis was post-thrombotic syndrome of both lower extremities and Behcet's disease. The patient was treated with medications such as "thalidomide, diclofenac, hydroxychloroquine sulfate and prednisone", while the dose of rivaroxaban was reduced to $15 \mathrm{mg}$ once a day. The patient's oral ulcers healed. Rashes on the legs improved compared to before the targeted treatment, redness and swelling subsided (Figure 1). There was also no longer obvious swelling or discomfort in the calves. In February 2019, a repeat of the colour Doppler ultrasound showed recanalization of the deep veins in the right lower limb and D-dimer was $87.87 \mathrm{ng} / \mathrm{ml}$, a significant reduction compared to the test in July 2018.

\section{Comment}

Behcet's disease, also known as Behcet's syndrome, was first reported by Turkish doctor Hulusi Behcet in 1937. It is a chronic systemic vascular inflammatory disease involving multiple systems and organs and is associated with diverse and complex clinical manifestations. According to which organ system is affected, the condition can be divided into vascular, gastrointestinal, and neurological types. The most prominent features of the condition include oral ulcers, genital ulcers, uveitis, and skin lesions [1]. Since the incidence of this disease is higher in Asian countries than Western countries, particularly in those along the old silk trading routes such as Turkey, Iran, China, and Japan, Behcet's disease is also known as "Silk Road Disease". The diagnosis of Behcet's disease is based mainly on clinical symptoms. As such, it is important to obtain detailed history and pay special attention to typical clinical signs and symptoms. The presence of damage to large blood vessels or to the nervous system is highly suggestive of the diagnosis of Behcet's disease [1,2].
At present, the diagnostic criteria for Behcet's disease were proposed by the International Study Group for Behcet's Disease in 1989 , which were as follows: repeated oral ulcers are a necessary condition, along with two or more out of the following four clinical features: recurrent genital ulcers, eye lesions, skin lesions, and a positive pathergy response [3]. Other signs and symptoms that are highly associated with a diagnosis of the disease include gastrointestinal ulcers, joint pain, and epididymal inflammation. In the case presented in this report, there were recurrent oral ulcers, genital ulcers, and eye lesions combined with skin lesions, vascular involvement, and joint pain, which were all indicative of Behcet's disease. At the same time, managing the condition as a case of Behcet's disease produced better treatment outcomes. The exact aetiology and pathogenesis of Behcet's disease are unknown. Its pathogenesis may be related to hereditary immune disorders, pathogen infections, or vitamin D deficiency. Immune cells, inflammatory factors and mediators have important regulatory roles in the pathogenesis, development, and prognosis of Behcet's disease [1].

In the literature, around 7\% - 46\% of patients with Behcet's disease have cardiovascular system involvement [1]. Behcet's disease associated with vascular lesions in large vessels is called Vasculo-Behcet's disease (VBD). Venous involvement often presents as superficial thrombophlebitis and deep venous thrombosis [4]. Compared to primary deep vein thrombosis, Behcet's disease with deep vein thrombosis is associated with a wider range of lesions, which is poor in outcomes and common in recurrence, often resulting in post-thrombotic syndrome [2]. Post-thrombotic syndrome is characterised by severe discomfort in the lower extremities that is exacerbated by prolonged standing or activity, as well as significant tenderness in the affected limb, intermittent claudication, limb swelling, increased muscle tone, superficial vein dilatation, pigmentation in the calf and foot, thickened and rough skin, itching, eczema-like dermatitis, long-standing chronic ulcer, etc.

Clinical manifestations of vena cava thrombosis are different depending on which part of the blood vessel is affected. Regarding the treatment of Behcet's disease with deep venous thrombosis in the lower extremities, compared with anticoagulants alone, the addition of immunosuppressants can reduce the recurrence of DVT in patients with Behcet's disease and deep vein thrombosis. Glucocorticoids combined with immunosuppressants such as cyclosporine A, azathioprine (AZA) or cyclophosphamide were recommended. It has been shown that statins can benefit patients with Behcet's disease by protecting vascular endothelial cell function [1]. The clinical manifestations of Behcet's disease are diverse and complex. Due to factors such as insufficient understanding of the disease as well as its low incidence, it is easy to overlook the diagnosis and treatment of the primary condition $[4,5]$.

When clinicians are faced with the common presentation of deep vein thrombosis in the lower limbs, the patient's past 
history should be paid attention to and a thorough examination is recommended if the cause of thrombosis is unknown, and there is history of skin lesions, long-term oral ulcers, and a poor response to anticoagulant treatment. Thrombophilia should be investigated, and a biopsy of skin lesions carried out. A comprehensive analysis, appropriate consideration of this condition, and a review by rheumatology/immunology specialists are required to avoid a missed or incorrect diagnosis.

\section{Conclusion}

In this case report we demonstrated that BD combined with LEDVT can lead to reduced efficacy of the anticoagulation therapy against LEDVT and inclination to relapse. Early discovery and treatment of BD can improve the treatment effect of LEDVT. Besides, the possibility of concurrent BD should be considered in those with LEDVT to reduce missed diagnosis and missed treatment.

ISSN: 2574-1241

DOI: $10.26717 /$ BJSTR.2020.31.005073

Hong Tao Xiong. Biomed J Sci \& Tech Res

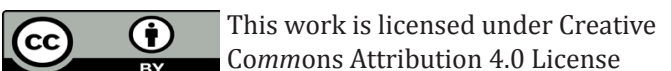

Submission Link: https://biomedres.us/submit-manuscript.php

\section{References}

1. Davatchi F, Chams Davatchi C, Shams H, Hoda Kavosi, Mariam Masoumi, et al. (2017) Behcet's disease: epidemiology, clinical manifestations, and diagnosis. Expert Rev Clin Immunol 13(1): 57-65.

2. Seyahi E, Cakmak OS, Tutar B, Melike Melikoglu, Hasan Yazici, et al. (2015) Clinical and ultrasonographic evaluation of lower-extremity vein thrombosis in Behcet syndrome: an observational study. Medicine 94(44): e1899.

3. Toledo Samaniego N, Galeano Valle F, Pinilla Llorente B (2019) Clinical features and Management of venous thromboembolism in patients with Behçet's syndrome: a single center case-control study. Intern Emerg Med 4: 1-10.

4. Seyahi E (2016) Behçet's disease: How to diagnose and treat vascular involvement. Best Pract Res Clin Rheumatol 30(2): 279-295.

5. Wu X, Li G, Huang X, Wang L, Wenjie Zheng, et al. (2014) Behçet's Disease Complicated with Thrombosis: A Report of 93 Chinese Cases. Medicine 93(28): e263.

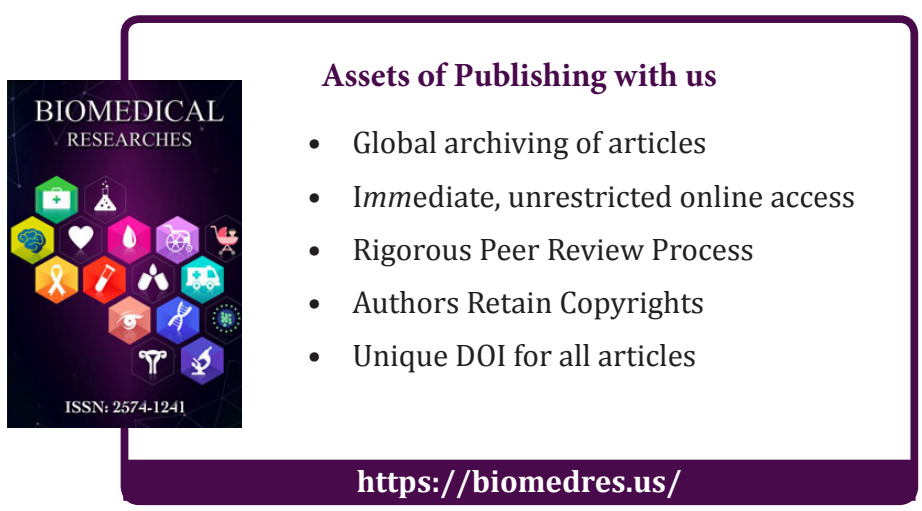

